\title{
EXISTENCE AND DIFFERENTIABILITY THEOREMS FOR THE SOLUTIONS OF VARIATIONAL PROBLEMS FOR MULTIPLE INTEGRALS ${ }^{1}$
}

\author{
CHARLES B. MORREY, JR.
}

\section{INTRODUCTION}

1. The object of the research [1] with which this address is concerned is twofold: first, to demonstrate, by direct methods, the existence of solutions, perhaps in some generalized sense, for a wide class of variational problems for multiple integrals, and second, to investigate further differentiability properties of the generalized solutions thus obtained. We consider only problems in nonparametric form.

2. Both of these goals have been achieved in the recent well known solutions of the problem of Plateau and in the solutions of the Dirichlet problem by variational methods. In more general problems this program has not been carried through completely except in very restricted cases. However, there are very important known results in connection with each separate aim. In connection with the existence theory, very important work has been done in the nonparametric case by Tonelli [2] and in the parametric case by McShane [3]. In the parametric case, practically nothing is known concerning the differentiability of the solutions obtained. In the nonparametric case, it has been proved by E. Hopf [4] that if $f(x, y, z, p, q)$ is of class $C_{\alpha}^{\prime \prime}$ (that is, if $f$ is of class $C^{\prime \prime}$ and its second derivatives satisfy a uniform Hölder condition ${ }^{2}$ with exponent $\alpha, 0<\alpha<1$, on any bounded portion of space) and is the integrand in a regular variational problem (that is, if $f_{p p} f_{q q}-f_{p q}^{2}>0, f_{p p}>0$ ) and if $z_{0}$ is continuous on $\bar{G}$ and is of class $C_{\beta}^{\prime}$ on each region $D$ with $\bar{D} \subset G$ and minimizes $\iint_{G} f(x, y, z, p, q) d x d y$ among all functions, continuous on $\bar{G}$, of class $C^{\prime}$ in $G$, and coinciding with $z_{0}$ on $G^{*}$, then $z_{0}$ is of class $C_{\gamma}^{\prime \prime}$ on any region $D$ as above for any $\gamma<\alpha$. The author has proved in a previous paper [5] that the same result holds if $z_{0}$ merely satisfies a uniform Lipschitz condition $^{3}$ on regions $\bar{D}$ interior to $G$. The result of $\mathrm{E}$. Hopf

1 An address delivered before the meeting of the Society in Pasadena on December 2, 1939, by invitation of the Program Committee.

${ }^{2}$ A function $f(P)$ is said to satisfy a uniform Hölder condition on a set $S$ if $\left|f\left(P_{1}\right)-f\left(P_{2}\right)\right| \leqq C \cdot\left|P_{1} P_{2}\right| \alpha, 0<\alpha<1$, for each pair of points $\left(P_{1}, P_{2}\right)$ in $S ; C$ is called the coefficient and $\alpha$ is called the exponent of the Hölder condition and $\left|P_{1} P_{2}\right|$ denotes the distance from $P_{1}$ to $P_{2} ; C$ and $\alpha$ are supposed to be independent of $P_{1}$ and $P_{2}$.

${ }^{3}$ A function $f(P)$ is said to satisfy a uniform Lipschitz condition on a set $S$ if $\left|f\left(P_{1}\right)-f\left(P_{2}\right)\right| \leqq C \cdot\left|P_{1} P_{2}\right|$ for every pair $\left(P_{1}, P_{2}\right)$ on $S, C$ independent of $P_{1}, P_{2}$. 
can be extended readily to the case where $f$ is a function of $\left(x, y, z, \cdots, z_{N}\right)$ while the methods used in the author's previous paper [5] do not permit this extension.

3 . In connection with the existence theory, Tonelli proves in the two papers mentioned that if $f(x, y, z, p, q)$ satisfies certain continuity restrictions, gives rise to a quasi-regular variational problem, and if it satisfies

$$
f(x, y, z, p, q) \geqq m\left(p^{2}+q^{2}\right)^{\alpha / 2}+N, \quad m>0, \alpha>2,
$$

or (essentially)

$$
f(x, y, z, p, q) \geqq m\left(p^{2}+q^{2}\right), \quad f(x, y, z, 0,0)=0, \quad m>0,
$$

then the integral

$$
I(z, G)=\iint_{G} f(x, y, z, p, q) d x d y
$$

takes on its minimum among all functions $z$ which are absolutely continuous in his sense and assume given continuous boundary values, provided $I(z, G)$ is finite for some such function and $G$ is properly restricted; more general boundary value problems are also considered. The proof consists in showing first that $I(z, G)$ is lower semicontinuous with respect to uniform convergence in his class, and then in replacing any minimizing sequence by one in which the functions are equicontinuous so that a uniformly convergent minimizing subsequence is obtained which can be shown to converge to a function in his class. If the $\alpha$ in (3.1) is less than 2, such a procedure is not possible in general. In fact, when we try to generalize these results to functions of $N$ independent variables, we find that Tonelli's procedure is impossible unless $\alpha \geqq N$ (see $\S 12$ ). As the gap $1<\alpha<N$ grows wider as $N$ increases, it seems desirable to obtain existence theorems of some sort assuming merely that $\alpha>1$; for $\alpha=1$, there are examples, even for $N=1$, of problems which have no solutions.

Evidently we must abandon the use of continuity and equicontinuity in obtaining our existence theorems but must certainly retain absolute continuity in some sense. G. C. Evans [6] has introduced a class of functions, which he calls "potential functions of their generalized derivatives," which would seem to form a proper class of admissible functions; we shall call these functions "functions of class $\mathfrak{B}$ " (see below). Surprisingly enough, these functions were introduced before those of Tonelli, and Evans [7] has investigated the connection between the two classes of functions (we state the results below). 
4. Before proceeding to a discussion of these functions and their applications, we must introduce certain notations and terminology. The $N$-tuples $\left(x_{1}, \cdots, x_{N}\right),\left(\xi_{1}, \cdots, \xi_{N}\right), \cdots$, will be denoted by the single letters $x, \xi, \cdots$, and the closed cell $a_{i} \leqq x_{i} \leqq b_{i}, i=1, \cdots, N$, will be denoted by $[a, b]$. The functional notation $f\left(x_{1}, \cdots, x_{N}\right)$ will be abbreviated to $f(x)$ and the Lebesgue integrals of $f(x)$ over $[a, b]$ or a set $E$ will be denoted by

$$
\int_{a}^{b} f(x) d x, \quad \int_{E} f(x) d x .
$$

It is frequently desirable to consider the behavior of a function $f(x)$ with respect to a particular coordinate variable $x_{k}$ or with respect to the $(N-1)$ variables $\left(x_{1}, \cdots, x_{k-1}, x_{k+1}, \cdots, x_{N}\right)$. We then denote the latter variables by $x_{k}^{\prime}$, we write $\left(x_{k}^{\prime}, x_{k}\right)$ for $x$ and $f\left(x_{k}^{\prime}, x_{k}\right)$ for $f(x)$ : thus $f\left(a_{k}^{\prime}, x_{k}\right)$ denotes the function $f\left(a_{1}, \cdots, a_{k-1}, x_{k}, a_{k+1}, \cdots, a_{N}\right)$ of the single variable $x_{k}\left(a_{k}^{\prime}\right.$ being fixed) and $f\left(x_{k}^{\prime}, a_{k}\right)$ denotes the function $f\left(x_{1}, \cdots, x_{k-1}, a_{k}, x_{k+1}, \cdots, x_{N}\right)$ ( $a_{k}$ being fixed). The projection of the cell $[a, b]$ on $x_{k}=0$ is denoted by $\left[a_{k}^{\prime}, b_{k}^{\prime}\right]$ and

$$
\int_{a_{k}^{\prime}}^{b_{k}^{\prime}} f\left(x_{k}^{\prime}, a_{k}\right) d x_{k}^{\prime}
$$

denotes the $(N-1)$-dimensional integral of $f\left(x_{k}^{\prime}, a_{k}\right)$ over $\left[a_{k}^{\prime}, b_{k}^{\prime}\right]$. If $G$ is a region (open connected set), $G^{*}$ denotes its boundary and $\bar{G}$ its closure. All integrals are Lebesgue integrals.

\section{The admissible functions}

5. We now define the various classes of functions of which we shall speak.

Definition 5.1. A function $f(x)$ is of class $\mathfrak{B}$ on a region $G$ if

(i) it is summable on each closed cell interior to $G$, and

(ii) there exist functions $v_{1}(x), \cdots, v_{N}(x)$, satisfying (i) and such that

$$
\int_{a_{k}^{\prime}}^{b_{k}^{\prime}}\left[f\left(x_{k}^{\prime}, b_{k}\right)-f\left(x_{k}^{\prime}, a_{k}\right)\right] d x_{k}^{\prime}=\int_{a}^{b} v_{k}(x) d x
$$

for almost all cells $[a, b]$ interior to $G$ (that is, all such cells such that the point $\left(a_{1}, \cdots, a_{N} ; b_{1}, \cdots, b_{N}\right)$ does not belong to a set of $2 N$-dimensional measure zero).

Definition 5.2. A function $f(x)$ is of class $\mathfrak{P}^{\prime}$ on $G$ if

(i) it satisfies (i) of Definition 5.1, and 
(ii) if $[a, b]$ is any cell interior to $G, f\left(x_{k}^{\prime}, x_{k}\right)$ is A.C. (absolutely continuous) in $x_{k}$ for $a_{k} \leqq x_{k} \leqq b_{k}$ for almost every $x_{k}^{\prime}$ in $\left[a_{k}^{\prime}, b_{k}^{\prime}\right]$, and

(iii) $\partial f / \partial x_{k}$ (which exists almost everywhere and is measurable by (i) and (ii)) is summable on each cell interior to $G$.

Definition 5.3 [8]. A function $f(x)$ is absolutely continuous in the sense of Tonelli (A.C.T.) on $G$ if

(i) it is continuous on $G$,

(ii) it satisfies (ii) of Definition 5.2, and

(iii) if $[a, b]$ is any cell in $G$, then the variation of $f\left(x_{k}^{\prime}, x_{k}\right)$ as a function of $x_{k}$ alone on $\left[a_{k}, b_{k}\right]$ is a summable (known to be lower semicontinuous from (i)) function of $x_{k}^{\prime}$.

Definition 5.4. A function $f(x)$ is of class $\mathfrak{B}^{\prime \prime}$ on $G$ if it is of class $\mathfrak{P}$ there and is continuous.

It is easily shown that a function of $f(x)$ of class $\mathfrak{B}$ determines the functions $v_{k}(x)$ of Definition 5.1 to within a null function (a function which vanishes almost everywhere). Hence it defines completely the set functions $\int_{e} v_{k}(x) d x(k=1, \cdots, N)$ which are A.C. on any bounded set $E$ whose closure is in $G$. Accordingly we propose, with Evans [6], the following definition:

Definition 5.5. The generalized derivative $D_{x_{k}} f$ of $f$ with respect to $x_{k}$ is defined as the Lebesgue derivative [9] of the set function $\int_{e} v_{k}(x) d x d e-$ fined above, wherever this derivative exists.

It is well known that this derivative exists almost everywhere and coincides with $v_{k}(x)$ almost everywhere. For simplicity, we shall use the notation $f_{x k}$ to denote either $D_{x k} f$ or $\partial f / \partial x_{k}$ as desired (see $\S 6$ for a justification of this usage).

6. The following theorems concerning the above classes of functions are easily demonstrated:

THEOREM 6.1. If $f(x)$ is of class $\mathfrak{B}$ on a region $G$, any function $f^{*}(x)$ which is equivalent to $f(x)$ on $G$ (that is, differs from $f(x)$ by a null function) is also of class $\mathfrak{B}$ on $G$ and the generalized derivatives of $f$ and $f^{*}$ exist at exactly the same points and coincide at these points.

Theorem 6.2. Any two functions of class $\mathfrak{P}$ whose respective generalized derivatives coincide almost everywhere differ at most by a constant and a null function.

TheOREM 6.3. Any function of class $\mathfrak{P}^{\prime}$ is of class $\mathfrak{P}$ and corresponding generalized and partial derivatives coincide almost everywhere. 
Theorem 6.4. Any function of class $\mathfrak{B}$ is equivalent to a function of class $\mathfrak{B}^{\prime}$.

Theorem 6.5. The classes in Definitions 5.3 and 5.4 coincide.

Theorem 6.6. A necessary and sufficient condition that $f(x)$ be of class $\mathfrak{P}$ on $G$ is that it satisfy (i) of Definition 5.1 and that for each cell $[a, b]$ interior to $G$, there exist summable functions $v_{k}(x)$ and a sequence $\left\{f_{n}(x)\right\}$, each $f_{n}$ satisfying a uniform Lipschitz condition on $[a, b]$, such that

$$
\lim _{n \rightarrow \infty} \int_{a}^{b}\left[\left|f_{n}-f\right|+\sum_{k=1}^{N}\left|f_{n, x_{k}}-v_{k}\right|\right] d x=0 .
$$

7. We now define the most important of all of our classes of admissible functions. The theorem below and those in the next five sections would make it appear that these functions should play an important rôle in many other branches of analysis as well as in the present research.

Definition 7.1. A function $f(x)$ is said to be of class $\mathfrak{B}_{\alpha}\left(\mathfrak{B}_{\alpha}^{\prime}\right.$ or $\left.\mathfrak{B}_{\alpha}^{\prime \prime}\right)$, $\alpha \geqq 1$, on $G$ if it is of class $\mathfrak{B}\left(\mathfrak{P}^{\prime}\right.$ or $\left.\mathfrak{B}^{\prime \prime}\right)$ there and if $|f|^{\alpha}$ and $\left|D_{x k} f\right|^{\alpha}$ are summable over $G, k=1, \cdots, N$.

The following theorem concerning these functions is of fundamental importance.

THEOREM 7.1. The space $\mathfrak{B}_{\alpha}$ of classes of equivalent functions of class $\mathfrak{P}_{\alpha}$ on a region $G$ is a Banach space (space of type B) [10] if we define, for instance,

$$
\begin{aligned}
\|f\|^{\alpha}=\bar{D}_{\alpha}(f, G) & \equiv D_{\alpha}(f, G)+\int_{G}|f|^{\alpha} d x, \\
D_{\alpha}(f, G) & \equiv \int_{G}\left[\sum_{i=1}^{N}\left|f_{x_{i}}\right|^{2}\right]^{\alpha / 2} d x,
\end{aligned}
$$

$\|f\|$ denoting the norm of $f$ in $\mathfrak{B}_{\alpha}$ on $G$.

8. Before proceeding further with the discussion of these functions, we propose two more definitions as follows:

Definition 8.1. A transformation $x=x(y)$ of a set $T$ into a set $S$ is Lipschitzian if it is 1-1 and continuous and if the functions $x_{i}(y)$ and $y_{i}(x)$ (of the inverse) all satisfy uniform Lipschitz conditions on each closed subset of $T$ and $S$ respectively. If the Lipschitz conditions are uniform over the whole of $T$ and $S$, we say that the Lipschitzian transformation is regular. 
Definition 8.2. A region $G$ is said to be Lipschitzian if $\bar{G}$ can be covered by a finite number of sets $\gamma_{1}, \cdots, \gamma_{L}$, each of which is the product of $\bar{G}$ with an open set and is the image under a regular Lipschitzian transformation of either the open unit cube $\left|y_{i}\right|<1$ or the part of this cube for which $y_{N} \leqq 0$; in the latter case we assume that the transformation sets up a 1-1 correspondence between the points of $\gamma_{i} \cdot G^{*}$ and the points of the half-cube for which $y_{N}=0$.

9. Rademacher [11] has shown that a function satisfying a Lipschitz condition on a region $G$ possesses a total differential almost everywhere and has also proved the usual theorems on transformations of multiple integrals to hold for Lipschitzian transformations. With these definitions and results in mind, we may generalize a result of Evans [12] as follows:

THEOREM 9.1. Let $f(x)$ be of class $\mathfrak{P}$ (or $\mathfrak{P}^{\prime \prime}$ ) on a region $G$ and let $x=x(y)$ be a Lipschitzian transformation of a region $H$ into $G$. Then the transformed function $f[x(y)]=g(y)$ is of class $\mathfrak{B}$ (or $\mathfrak{B}^{\prime \prime}$ ) on $H$. Moreover if $x_{0}=x\left(y_{0}\right)$ where $y_{0}$ is a point of $H$ at which all the $x_{i}(y)$ possess total differentials and if all the generalized derivatives of $f$ exist at $x_{0}$, then all the generalized derivatives of $g$ exist at $y_{0}$ and are connected with those of $f$ at $x_{0}$ by the usual formulas. If $f$ is of class $\mathfrak{B}_{\alpha}$ on $G$ and the transformation is regular, then $g$ is of class $\mathfrak{B}_{\alpha}$ on $H$.

Concerning the functions of class $\mathfrak{P}^{\prime}$, we have proved the following theorem:

TheOREM 9.2. Any function of class $\mathfrak{B}$ on $G$ is equivalent to a function of class $\mathfrak{B}^{\prime}$ on $G$ which is transformed into a function of class $\mathfrak{B}^{\prime}$ by any regular Lipschitzian transformation.

Not every function of class $\mathfrak{B}^{\prime}$ has this property, and even if it does, an example of Saks [13] shows that the statement of Evans concerning the generalized derivatives is not in general true for the partial derivatives.

10. We now come to a discussion of the boundary values of functions of class $\mathfrak{P}_{\alpha}$ on bounded regions. If $f(x)$ is of class $\mathfrak{B}_{\alpha}$ on a Lipschitzian region $G(\alpha \geqq 1)$, we may state the following fundamental results:

THEOREM 10.1. There exists a sequence $\left\{f_{n}(x)\right\}$ of functions, each satisfying a uniform Lipschitz condition on $\bar{G}$, which converges strongly, accordingly to the norm (7.1) to $f$ on $G$.

THEOREM 10.2. There exists a boundary value function $f^{*}$ of class $L_{\alpha}$ 
on $G^{*}$ (with respect to hyper-area on $G^{*}$ ) such that $f_{n}$ converges strongly in $L_{\alpha}$ on $G^{*}$ to $f^{*},\left\{f_{n}\right\}$ being any sequence as in Theorem 10.1 .

THEOREM 10.3. If $f^{*}=0$ almost everywhere, the sequence $\left\{f_{n}(x)\right\}$ of Theorem 10.1 may be chosen so that each $f_{n}$ vanishes on and near $G^{*}$.

TheOREM 10.4. If $x^{\prime}=x^{\prime}(x)$ is a regular Lipschitzian transformation of $G$ into $G^{\prime}$ and if $f^{\prime}$ and $f^{\prime *}$ are the transforms of $f$ and $f^{*}$, then $f^{* *}$ is the boundary value function for $f^{\prime}$ on $G^{\prime *}$.

Theorem 10.5. Let $\bar{f}(x)$ be of class $\mathfrak{B}_{\alpha}^{\prime}$ and equivalent to $f(x)$ on $G$. Then there exist sets $Z_{k}, k=1, \cdots, N$, each of $(N-1)$-dimensional measure zero, such that if $\bar{x}_{k}^{\prime}$ is not in $Z_{k}, \bar{f}\left(\bar{x}_{k}^{\prime}, x_{k}\right)$ is $A . C$. on each segment $x_{k}^{(1)} \leqq x_{k} \leqq x_{k}^{(2)}$, such that $\left(\bar{x}_{k}^{\prime}, x_{k}\right)$ is in $G$ and $\left(\bar{x}_{k}^{\prime}, x_{k}^{(1)}\right)$ is on $G^{*}$, $i=1,2$, and $\bar{f}\left(\bar{x}_{k}^{\prime}, x_{k}\right)$ tends to $f^{*}\left(\bar{x}_{k}^{\prime}, x_{k}^{(i)}\right)$ as $x_{k}$ tends to $x_{k}^{(i)}$ along this segment, $i=1,2, k=1, \cdots, N$.

If $G$ is merely an arbitrary bounded region, we do not have such explicit results but we may follow a procedure suggested by Courant [14]. This procedure suggests the following definition:

Definition 10.1. A function $f(x)$ of class $\mathfrak{B}_{\alpha}$ on $G$ is said to vanish on $G^{*}$ if there exists a sequence $\left\{f_{n}(x)\right\}$ of functions, each satisfying a uniform Lipschitz condition on $\bar{G}$ and vanishing on and near $G^{*}$, such that $\left\|f_{n}-f\right\| \rightarrow 0$. Two functions $f_{1}$ and $f_{2}$ of class $\mathfrak{P}_{\alpha}$ are said to coincide on $G^{*}$ if their difference vanishes on $G^{*}$ in the above sense.

From Theorem 10.3, it is clear that the above definition of vanishing coincides with the condition that $f^{*}=0$ in the case that $G$ is Lipschitzian. Using this very general terminology, we may state the following very important "substitution theorem":

THEOREM 10.6. Let $f(x)$ be of class $\mathfrak{B}_{\alpha}$ on a region $G$, let $g(x)$ be of class $\mathfrak{P}_{\alpha}$ on a subregion $D$ of $G$ and coincide with $f(x)$ on $D^{*}$, and let $h(x)$ be defined equal to $f$ on $G-D$ and equal to $g$ on $D$. Then $h(x)$ is of class $\mathfrak{P}_{\alpha}$ on $G$, coincides with $f(x)$ on $G^{*}$, and $D_{x_{k}} h=D_{x_{k}} f$ almost everywhere on $G-D$ ( $D$ is open) and $D_{x_{k}} h=D_{x_{k}} g$ on $D$ wherever either exists, $k=1, \cdots, N$.

11. As the spaces $\mathfrak{B}_{\alpha}$ have been seen to be Banach spaces, we may inquire into the nature and consequences of weak convergence [15] in $\mathfrak{P}_{\alpha}$. In this connection, the following interesting results have been proved:

THEOREM 11.1. The most general linear functional [16] $F(f)$ defined on $\mathfrak{B}_{\alpha}$ is of the form 


$$
F(f)=\int_{G}\left[A_{0}(x) f(x)+\sum_{i=1}^{N} A_{i}(x) D_{x_{i}} f\right] d x
$$

where the $A_{i}$ are essentially bounded if $\alpha=1$ and are in $L_{\beta}$ with $\alpha^{-1}+\beta^{-1}=1$ if $\alpha>1$ (the $A_{i}(x)$ are not uniquely determined by $F(f)$ however).

TheOREM 11.2. A necessary and sufficient condition that $f_{n}$ tend weakly to $f$ in $\mathfrak{B}_{\alpha}$ is that $f_{n}$ and $D_{x_{i}} f_{n}$ tend weakly to $f$ and $D_{x_{i}} f$, respectively.

THEOREM 11.3. Weak convergence is preserved by regular Lipschitzian transformations.

THEOREM 11.4. If $f_{n}$ tends weakly to $f$ in $G$, the convergence holds on any subregion and $f$ is uniquely determined.

TheOREM 11.5. If the $f_{n}$ all vanish on $G^{*}, f$ vanishes on $G^{*}$.

THEOREM 11.6. If $G$ is Lipschitzian and $f_{n}$ tends weakly in $\mathfrak{B}_{\alpha}$ on $G$ to $f$, then $f_{n}$ tends strongly in $L_{\alpha}$ to $f$ on $G$ and the boundary value functions $f_{n}^{*}$ tend strongly in $L_{\alpha}$ on $G^{*}$ to $f^{*}$.

THEOREM 11.7. If $\alpha>1, a$ necessary and sufficient condition that a family of functions $\{f\}$ in $\mathfrak{B}_{\alpha}$ be compact (perhaps not closed) with respect to weak convergence in $\mathfrak{P}_{\alpha}$ is that their norms be uniformly bounded. If $\alpha=1$ we must also have a convex function $\phi\left(p_{1}, \cdots, p_{N}\right)$ with

$$
\lim _{|p| \rightarrow \infty} \phi\left(p_{1}, \cdots, p_{N}\right) /|p|=+\infty, \quad|p|^{2}=p_{1}^{2}+\cdots+p_{N}^{2},
$$

such that

$$
\int_{G} \phi\left[D_{x_{1}} f, \cdots, D_{x_{N}} f\right] d x
$$

is uniformly bounded.

THEOREM 11.8. A necessary and sufficient condition that the norms in $\mathfrak{P}_{\alpha}$ of each function of a family be uniformly bounded is that $D_{\alpha}(f, G)$ be uniformly bounded, each function $f$ of the family coinciding on $G^{*}$ with a function $g$ of another family whose norms are uniformly bounded.

THEOREM 11.9. If $G$ is Lipschitzian, the above condition may be replaced by either the condition that $\bar{D}_{\alpha}(f, \tau)$ be uniformly bounded for some cell $\tau$ in $G$ or the condition that $\int_{E}\left|f^{*}\right|{ }^{\alpha} d s(e)$ be uniformly bounded, $E$ being some set open on $G^{*}$ and $s(e)$ being the hyper-area set function on $G^{*}$. 
12. Of less interest here are Green's theorem for functions of class $\Re_{1}$ on a Lipschitzian region $G$ and theorems hinted at above (in $\S 3$ ) which show that functions of class $\mathfrak{B}_{\alpha}$ with $\alpha>N$ are equivalent to continuous functions. If $\alpha \leqq N$, then this is not usually true as the examples

$$
\begin{array}{lrl}
f(x)=\log \log \left(1+r^{-1}\right), & \alpha=N, r^{2}=x_{1}^{2}+\cdots+x_{N}^{2}, \\
f(x)=r^{-\beta}, & \alpha<N, 0<\beta, \alpha(\beta+1)<N,
\end{array}
$$

show.

III. THE EXISTENCE THEORY

13. Having studied our admissible functions at some length, we can now easily derive some very general existence theorems. We consider integrals

$$
\begin{array}{rlrl}
I(z, G) & =\int_{G} f\left(x_{1}, \cdots, x_{N}, z_{1}, \cdots, z_{P}, p_{1}^{1}, \cdots, p_{N}^{1}, \cdots, p_{1}^{P}, \cdots, p_{N}^{P}\right) d x \\
& =\int_{G} f(x, z, p) d x, & p_{j}^{i}=D_{x_{j} z_{i}},
\end{array}
$$

in which $f(x, z, p)$ is continuous all over the space of its arguments, is convex in $p$ for each $(x, z)$, and satisfies a condition

$$
f(x, z, p) \geqq \phi(p), \quad \lim _{|p| \rightarrow \infty}|p|^{-1} \phi(p)=+\infty, \quad|p|^{2}=\sum_{i=1}^{N} \sum_{j=1}^{P}\left(p_{i}^{j}\right)^{2},
$$

$\phi(p)$ being convex. It is straightforward to prove that such an integral $I(z, G)$ is lower semicontinuous with respect to weak convergence in $\mathfrak{P}_{1}$. From our study of the admissible functions, we see that any family of functions $z$ for which $I(z, G)$ is uniformly bounded will be compact with respect to weak convergence in $\mathfrak{P}_{1}$, provided merely that the norms are uniformly bounded. This may be ensured by satisfying one of the conditions in Theorems 11.8 or 11.9. If this can all be done for a minimizing sequence, we may then pick out a subsequence which converges weakly in $\mathfrak{P}_{1}$ to some function which is also in $\mathfrak{P}_{1}$. From the lower semicontinuity of $I(z, G)$ we may conclude that our limit function is a minimizing function. It is clear that a great variety of existence theorems with variable as well as fixed boundary values can be proved with very little difficulty. For example, we may conclude the existence of a minimal surface part of whose boundary is to be a fixed Jordan arc or is to lie on a bounded closed manifold. Of course our existence theorems do not allow us to conclude continuity 
of the surface on the boundary and, indeed, this is quite difficult to prove and may not be true in many problems.

\section{Continuity PRoperties of the Minimizing FUnCtions}

14. Without placing further restrictions on the integrand $f(x, z, p)$, one cannot expect to be able to deduce many further properties of the solutions obtained from the above existence theory. This type of study seems quite difficult and is almost unexplored. Accordingly we have restricted ourselves to the case $N=2$ and have required that our function ( $N$ is the previous $P$ )

$$
f\left(x, y, z^{1}, \cdots, z^{N}, p^{1}, \cdots, p^{N}, q^{1}, \cdots, q^{N}\right)=f(x, y, z, p, q)
$$

be of class $C_{\alpha}^{\prime \prime}$ over the space of its arguments and that it satisfy the further conditions

$$
\begin{aligned}
& m_{1}\left(p^{2}+q^{2}\right) \leqq f(x, y, z, p, q) \leqq M_{1}\left(p^{2}+q^{2}\right), \\
& p^{2}+q^{2}=\sum_{i=1}^{N}\left[\left(p^{i}\right)^{2}+\left(q^{i}\right)^{2}\right], 0<m_{1} \leqq M_{1}, \\
& m_{2}(R)\left(\xi^{2}+\eta^{2}\right) \leqq f_{p^{\alpha} p \beta} \xi^{\alpha} \xi^{\beta}+2 f_{p^{\alpha} q} \beta \xi^{\alpha} \eta^{\beta}+f_{q^{\alpha} q} \beta \eta^{\alpha} \eta^{\beta} \\
& \leqq M_{2}(R)\left(\xi^{2}+\eta^{2}\right), \xi^{2}+\eta^{2}=\sum_{i=1}^{N}\left[\left(\xi^{i}\right)^{2}+\left(\eta^{i}\right)^{2}\right], \\
& \sum_{i=1}^{N}\left[f_{p^{i} x}^{2}+f_{p^{i} y}^{2}+f_{q^{i} x}^{2}+f_{q^{i} y}^{2}+\left|f_{z i x}\right|+\left|f_{z i y}\right|\right. \\
&\left.\quad+\sum_{j=1}^{N}\left\{f_{p^{i z z i}}^{2}+f_{q^{i} z i}^{2}+\left|f_{z i z i}\right|\right\}\right] \leqq M_{3}(R)\left(p^{2}+q^{2}\right), \\
& 0<m_{2}(R) \leqq M_{2}(R), \quad x^{2}+y^{2}+\left(z^{1}\right)^{2}+\cdots+\left(z^{N}\right)^{2} \leqq R^{2},
\end{aligned}
$$

where in (14.1) we assume $m_{1}$ and $M_{1}$ to be independent of $(x, y, z, p, q)$ and where $m_{2}(R), M_{2}(R)$, and $M_{3}(R)$ depend only on $R$.

This type of integrand, although quite restricted, is not trivial. For instance, the integrand obtained in the problem of Plateau on a Riemannian manifold (if Courant's method [17] for the ordinary problem of Plateau is used) is

$$
g_{\alpha \beta}\left(z^{1}, \cdots, z^{N}\right)\left(p^{\alpha} p^{\beta}+q^{\alpha} q^{\beta}\right),
$$

which satisfies all the conditions (14.1) and (14.2) on the cell of the $z$ space in which the $g_{i j}(z)$ are defined, provided merely that the part of the manifold thus represented is regular and the $g_{i j}$ are of class $C_{\alpha}^{\prime \prime}$. Moreover, the existence theory for such an integrand does not follow 
from the results of Tonelli as the corresponding restrictions (3.2) for his methods to be extended to $N$ dependent variables $z^{1}, \cdots, z^{N}$ would be (essentially)

$$
\begin{gathered}
f(x, y, z, p, q) \geqq m\left(p^{2}+q^{2}\right), \quad m>0, \\
f\left(x, y, z^{1}, \cdots, z^{N}, p^{1}, \cdots, p^{k-1}, 0, p^{k+1}, \cdots, p^{N}, q^{1}, \cdots, q^{k-1},\right. \\
\left.0, q^{k+1}, \cdots, q^{N}\right)=0, \quad k=1, \cdots, N,
\end{gathered}
$$

such a set of equalities not being assumed in our case. In fact it seems very difficult to show the existence of solutions using methods which involve equicontinuity.

We have used the tensor summation convention in this section, summing only over repeated Greek indices, and we shall continue its use throughout the rest of the paper.

15. Of fundamental importance in the continuity and differentiability theory are the following four theorems:

THEOREM 15.1. If the vector function $z$ (that is, each component $z^{i}$, $i=1, \cdots, N)$ is of class $\mathfrak{P}_{2}$ on $G$ and if

$$
D_{2}[z, C(P, r)] \leqq M(r / a)^{\lambda}
$$

for each circle $C(P, r)$ in $G$, then $z$ (is equivalent to a function which) satisfies a uniform Hölder condition with exponent $\frac{1}{2} \lambda$ on each region $D$ with $\bar{D} \subset G$, the coefficient in which depends only on $M, \lambda$, and the distance of $\bar{D}$ from $G^{*} ;$ a denotes the distance of $P$ from $G^{*}$.

THEOREM 15.2. If, in Theorem 15.1, (15.1) holds only for circles with center at a fixed point $P$, then the average of $z$ over circles $C(P, r)$ tends to a limit $\bar{z}(P)$ as $r \rightarrow 0$. If $z$ vanishes on $G^{*}$, then

$$
|\bar{z}(P)| \leqq J(M, \lambda, a, \delta),
$$

where $J$ is a number depending only on the indicated quantities, $\delta$ being the diameter of $G$ and $a$ being the distance of $P$ from $G^{*}$.

THEOREM 15.3. Let $z$ be of class $\mathfrak{P}_{2}$ on $G$ and satisfy

$$
D_{2}(z, \Delta) \leqq K \cdot D_{2}[H(z, \Delta), \Delta], \quad K \geqq 1,
$$

for each region $\Delta$ in $G, H(z, \Delta)$ being the harmonic function in $\Delta$ coinciding with $z$ on $\Delta^{*}$ (known to exist by our existence theorems and easily proved harmonic). Suppose $G$ is bounded by a finite number of nonintersecting simple closed curves and suppose that $z$ coincides with a function of class $\mathfrak{B}_{2}^{\prime \prime}$ on $G$ and continuous on $\bar{G}$.

Then $z$ is continuous on $\bar{G}$ (or equivalent to such a function). 
THEOREM 15.4. If $z$ is of class $\mathfrak{P}_{2}$ on $G$ and satisfies

$$
\begin{array}{r}
D_{2}[z, C(P, r)] \leqq K \cdot D_{2}[H\{z, C(P, r)\}, C(P, r)]+L \cdot(r / a)^{\lambda}, \\
0<\lambda<K^{-1},
\end{array}
$$

for all circles in $G$ with center at a fixed point $P(0 \leqq r \leqq a)$, then $z$ satisfies (15.1), the $M$ of (15.1) depending only on $K, L$, and $\lambda$.

Some of these theorems have generalizations to vector functions of $n(n \geqq 2)$ variables. To generalize Theorems 15.1 and 15.2 , we need only to replace the $\lambda$ of (15.1) by $n-2+\lambda, \lambda>0$. Theorem 15.3 has no immediate generalization for all numbers $K$ and Theorem 15.4 yields an exponent $(n-1) / K$ which is not greater than $n-2$ unless $K$ is restricted to be quite near to unity. As we use Theorem 15.4 to obtain a condition (15.1), it will thus appear to the reader that the methods used in this research will not generalize immediately to integrands of the type described in $\$ 14$ where $x$ and $y$ are replaced by $n$ independent variables, essential use being made of the bound (15.2) and the continuity results of Theorem 15.1, both of which require the exponent $n-2+\lambda, \lambda>0$.

16. Now, if $z$ minimizes our integral $I(z, G)$ among all functions of class $\mathfrak{\beta}_{2}$ which coincide with it on $G^{*}$, then it follows from (14.1) that $z$ satisfies the condition in Theorem 15.3 with $K=M_{1} / m_{1}$; for if some $D$ exists where this does not hold, we can define $Z(x)=z(x)$ on $G-D$ and define $Z(x)=H(z, D)$ on $D$; then $Z(x)$ is of class $\mathfrak{P}_{2}$ on $G$ and we have (using Theorem 10.6)

$$
\begin{aligned}
I(Z, G) & =I(z, G-D)+I[H(z, D), D] \\
& \leqq I(z, G-D)+M_{1} D_{2}[H(z, D), D] \\
& <I(z, G-D)+m_{1} D_{2}(z, D) \leqq I(z, G),
\end{aligned}
$$

so that $z$ would not minimize $I(z, G)$. Thus, from the theorems in $\S 15$, we see that $D_{2}[z, C(P, r)] \leqq(r / a)^{\lambda} \cdot D_{2}(z, G), \lambda=m_{1} / M_{1}$, for each circle in $G$ with $r \leqq a$, that $z$ satisfies a uniform Hölder condition, with exponent $\frac{1}{2} \lambda$ on closed regions interior to $G$, and if the boundary values are continuous and $G$ is bounded by a finite number of simple closed curves, then $z$ is continuous on $\bar{G}$.

17. To proceed with the examination of our minimizing function $z$, we next can extend Haar's well known lemma [18] to show that $z$ satisfies

$$
\int_{R^{*}}\left(f_{p^{i}} d y-f_{q^{i}} d x\right)=\iint_{R} f_{z^{i}} d x d y
$$


for almost all rectangles $R$. If $D$ is any simply connected subregion of $G$, we may find functions $w^{i}$ and $W^{i}$ of class $\mathfrak{P}_{2}^{\prime \prime}$ which satisfy

$$
\begin{gathered}
w_{x}^{i}-W_{y}^{i}=-f_{q^{i}}, \quad w_{y}^{i}+W_{x}^{i}=f_{p^{i}}, \\
\int_{R^{*}}\left(W_{x}^{i} d y-W_{y}^{i} d x\right)=\iint_{R} f_{z^{i}} d x d y,
\end{gathered}
$$

almost everywhere and for almost all $R$ on $D$. We also note that if $D$ is any simply connected region with $\bar{D} \subset G$ and $z$ is any minimizing function for $I(z, G)$ (whether continuous on the boundary or not), then (referring to (14.2) and various lemmas in potential theory) it follows that

$$
\begin{aligned}
D_{2}[z, D \cdot C(P, r)] \leqq M_{4} r^{\lambda}, & D_{2}[w, D \cdot C(P, r)] \leqq M_{4} r^{\lambda}, \\
D_{2}[W, D \cdot C(P, r)] \leqq M_{4} r^{\lambda}, & \lambda>0,
\end{aligned}
$$

and (14.2) holds with $R=\max _{x, y \varepsilon D}\left[x^{2}+y^{2}+\left(z^{1}\right)^{2}+\cdots+\left(z^{N}\right)^{2}\right]$ which is bounded on such a region $\bar{D}$.

18. We next resort to a device due to Lichtenstein [19] and used by E. Hopf [4]. This consists in subtracting equation (17.1) formed for a rectangle $(a, c ; b, d)(a \leqq x \leqq b, c \leqq y \leqq d)$ from the same equation for the rectangle $(a+h, c ; b+h, d)$ or the rectangle $(a, c+h ; b, d+h)$ and then dividing by $h$. We confine ourselves to a region $\Delta$ such that $\bar{\Delta} \subset D \subset \bar{D} \subset G$. If we use the first rectangle above, we fińd that if $h$ is fixed and sufficiently small,

$$
\begin{aligned}
\int_{R^{*}} & \left(a_{i \beta} u_{x}^{\beta}+b_{i \beta} u_{y}^{\beta}+d_{i \beta} u^{\beta}+g_{i}\right) d y \\
& -\left(b_{\beta i} u_{x}^{\beta}+c_{i \beta} u_{y}^{\beta}+e_{i \beta} u^{\beta}+k_{i}\right) d x \\
& =\iint_{R}\left(d_{\beta i} u_{x}^{\beta}+e_{\beta i} u_{y}^{\beta}+f_{i \beta} u^{\beta}+l_{i}\right) d x d y, \quad i=1, \cdots, N,
\end{aligned}
$$

for almost all rectangles $R$ in $\Delta$ where the coefficients are measurable functions and $a_{i j}$, for instance, is given almost everywhere by the formula

$$
\begin{gathered}
a_{i j}(x, y)=\int_{0}^{1} f_{p^{i} p^{j}}\left[x+t h, y, z^{i}(x, y)+t\left\{z^{i}(x+h, y)-z^{i}(x, y)\right\}\right. \\
p^{i}(x, y)+t\left\{p^{i}(x+h, y)-p^{i}(x, y)\right\} \\
\left.q^{i}(x, y)+t\left\{q^{i}(x+h, y)-q^{i}(x, y)\right\}\right] d t
\end{gathered}
$$


and the other coefficients are given by similar formulas, and the $u^{i}(x, y)$ are the functions

$$
u^{i}(x, y)=\frac{z^{i}(x+h, y)-z^{i}(x, y)}{h}
$$

It is easily seen, using (14.2), (17.3), and (18.2) that there exists an $h_{0}>0$ and numbers $m, M, P$, and $\mu$ with $0<m \leqq M$ such that the coefficients satisfy (almost everywhere) the inequalities

$$
\begin{aligned}
& m\left(\xi^{2}+\eta^{2}\right) \leqq a_{\alpha \beta} \xi^{\alpha} \xi^{\beta}+2 b_{\alpha \beta} \xi^{\alpha} \eta^{\beta}+c_{\alpha \beta} \eta^{\alpha} \eta^{\beta} \leqq M\left(\xi^{2}+\eta^{2}\right), \\
& \iint_{\Delta \cdot C(P, r)} \sum_{i=1}^{N}\left[\left|l_{i}\right|+\sum_{j=1}^{N}\left\{d_{i j}^{2}+e_{i j}^{2}+\left|f_{i j}\right|\right\}\right] d x d y \leqq P r^{\mu} \\
& \iint_{\Delta \cdot C(P, r)}\left(g^{2}+k^{2}\right) d x d y \leqq P r^{\mu}, \quad \mu>0,
\end{aligned}
$$

for all circles $C(P, r)$ if $|h|<h_{0}$. By taking the rectangle $(a, c+h$; $b, d+h)$, we obtain similar equations and the same bounds as in (18.4) if $h_{0}$ is small enough, but in this case we have

$$
u^{i}(x, y)=\frac{z^{i}(x, y+h)-z^{i}(x, y)}{h}
$$

We emphasize that the bounds $m, M, P$, and $\mu$ are independent of $h$ if $|h|<h_{0}$. If $D$ is simply connected, we can carry out simultaneously a similar process on the equations (17.2) and we get

$$
\begin{aligned}
v_{x}^{i}-V_{y}^{i} & =-\left(b_{\beta i} u_{x}^{\beta}+c_{i \beta} u_{y}^{\beta}+e_{i \beta} u^{\beta}+k_{i}\right), \\
v_{y}^{i}+V_{x}^{i} & =a_{i \beta} u_{x}^{\beta}+b_{i \beta} u_{y}^{\beta}+d_{i \beta} u^{\beta}+g_{i}, \\
\int_{R^{*}} V_{x}^{i} d y-V_{y}^{i} d x & =\iint_{R}\left(d_{\beta i} u_{x}^{\beta}+e_{\beta i} u_{y}^{\beta}+f_{i \beta} u^{\beta}+l_{i}\right) d x d y, \\
v^{i}(x, y) & =\frac{w^{i}(x+h, y)-w^{i}(x, y)}{h}, \ldots, \ldots, N, \\
V^{i}(x, y) & =\frac{W^{i}(x+h, y)-W^{i}(x, y)}{h}, \ldots,
\end{aligned}
$$

almost everywhere and for almost all $R$. From (17.3) we have numbers $Q$ and $\nu$, independent of $h$ if $|h|<h_{0}$ such that 


$$
\iint_{\Delta \cdot C(P, r)}\left(u^{2}+v^{2}+V^{2}\right) d x d y \leqq Q r^{\nu}, \quad \nu>0
$$

for all $C(P, r)$.

19. In the course of this research, we have demonstrated the following theorem concerning the system (18.6):

THEOREM 19.1. Any solution ( $u, v, V)$ of (18.6) which satisfies (18.7) on a region $\Delta$ is of class $\mathfrak{P}_{2}^{\prime \prime}$ and satisfies a uniform Hölder condition on each region $H$ with $\bar{H} \subset \Delta$; the coefficient and exponent of this Hölder condition depend only on the diameter of $H$, the distance of $\bar{H}$ from $\Delta^{*}$, and on the numbers $m, M, P, \mu, Q$, and $\nu$ of $\$ 18$.

Thus if $H$ is any region with $\bar{H} \subset G$, we may let $h \rightarrow 0$ in $\$ 18$ and we then conclude (since we may then choose regions $\Delta$ and $D$ with $\bar{H} \subset \Delta \subset \bar{\Delta} \subset D \subset \bar{D} \subset G)$ that the first derivatives of $z$ satisfy uniform Hölder conditions on $\bar{H}$. This result plus that of E. Hopf mentioned in $\$ 2$ and those in $\$ 16$ lead us to the final theorem:

Theorem 19.2. If the integrand $f(x, y, z, p, q)$ of $\$ \$ 13$ and 14 satisfies the conditions of those two sections and if $z$ minimizes $I(z, G)$ among all functions of class $\mathfrak{P}_{2}$ on a region $G$ which coincide with $z$ on $G^{*}$, then $z$ is of class $C_{\beta}^{\prime \prime}$ on any region $H$ with $\bar{H} \subset G$ for each $\beta<\alpha, \alpha$ being the $\alpha$ of $\$ 14$. Moreover if $G$ is bounded by a finite number of simple closed curves and if the boundary values of $z$ are continuous on $G^{*}$, then $z$ is continuous on $\bar{G}$.

This result together with those of $\$ 13$ completes the program announced in $\$ 1$ for the important class of variational problems described in $\$ 14$.

It should be remarked that S. Bochner [20] has been able to solve the Euler differential equations for the integrand (14.3) (probably by means of the topological methods of Leray and Schauder [21]) and to prove certain properties of the solutions. He has been unable, however, to demonstrate the minimizing property of his solutions. The advantage of the procedure in this paper for this purpose is evident. By using direct methods, the author [22] has recently solved the Plateau problem (one contour case) on a Riemannian manifold of considerable generality, the surface thus obtained then being shown, using the results in this paper, to be of class $C^{\prime \prime}$.

\section{A study of The systems (18.1) AND (18.6)}

20. The most interesting part of this research, however, is concerned with the study of the systems (18.1) and (18.6) (which is 
equivalent to (18.1) if $\Delta$ is simply connected) which leads to the result stated in Theorem 19.1. Accordingly, we shall present an outline of this study.

It is easily seen that the equations (18.1), with $d_{i j}=e_{i j}=f_{i j}=l_{i}=0$ are the Haar equations for a function $u$ which minimizes the integral

$$
J(u, \Delta)=\iint_{\Delta}\left(a_{\alpha \beta} u_{x}^{\alpha} u_{x}^{\beta}+2 b_{\alpha \beta} u_{x}^{\alpha} u_{y}^{\beta}+c_{\alpha \beta} u_{y}^{\alpha} u_{y}^{\beta}+2 g_{\alpha} u_{x}^{\alpha}+2 k_{\alpha} u_{y}^{\alpha}+H\right) d x d y
$$

where $H$ is chosen summable and just large enough to make the integrand non-negative. It is also easy to show that $J(u, \Delta)$ is lower semicontinuous with respect to weak convergence in $\mathfrak{P}_{2}$ and that minimizing functions exist which coincide on $\Delta^{*}$ with any given function of class $\mathfrak{\beta}_{2}$. Also if a function $u_{0}$ of class $\mathfrak{P}_{2}$ on $\Delta$ satisfies (18.1) with $d_{i j}=e_{i j}=f_{i j}=l_{i}=0$, then it can easily be shown that it minimizes $J(u, \Delta)$ among all functions $u$ of class $\mathfrak{P}_{2}$ on $\Delta$ which coincide with $u_{0}$ on $\Delta^{*}$. If $g_{i}=k_{i}=0(i=1, \cdots, N)$, we may show as in (16.1) that a minimizing function $u_{0}$ satisfies (15.3) with $K=M / m$, so that the continuity restrictions obtained for $z$ in $\$ 16$ follow. If $g$ and $k$ are any functions of class $L_{2}$ (whether they satisfy (18.4) or not) the above minimizing argument is valid. If $g$ and $k$ satisfy

$$
\iint_{\Delta \cdot C(P, r)}\left(g^{2}+k^{2}\right) d x d y \leqq T r^{\pi}, \quad 0<\pi<\mu,
$$

for all circles with center at a point $P$ in $\Delta$, we conclude as in $\S 16$ that a solution $u_{0}$ satisfies a condition (15.4) and hence a condition (15.1) for circles with centers at $P$ and therefore obeys the conclusions of Theorem 15.2.

If $l_{i}$ satisfy (18.4), we may find potential functions

$$
V^{i}(x, y)=\frac{1}{4 \pi} \iint_{\Delta} \log \left[(\xi-x)^{2}+(\eta-y)^{2}\right] l_{i}(\xi, \eta) d \xi d \eta
$$

which can be shown to be of class $\mathfrak{P}_{2}^{\prime \prime}$ and to satisfy a condition (17.3) on any region in the plane and to satisfy

$$
\int_{R^{*}} V_{x}^{i} d y-V_{y}^{i} d x=\iint_{\Delta \cdot R} l_{i} d x d y
$$

for almost all rectangles $R$ in the plane. We then can solve (18.1) with given boundary values with only the $d_{i j}, e_{i j}$, and $f_{i j}=0$ by solving it with $l_{i}$ also zero and $g_{i}$ and $k_{i}$ replaced by $g_{i}-V_{x}^{i}$ and $k_{i}-V_{y}^{i}$. The solution is easily shown to be unique. 
21. We now let $G$ be an arbitrary bounded region and let $\mathfrak{B}_{2}$ denote the linear subspace of $\mathfrak{P}_{2}$ consisting of functions $u$ in $\mathfrak{P}_{2}$ which vanish on $G^{*}$. For such functions $u$, we define the linear operator $T u$ as the unique solution $U$ in $\mathfrak{B}_{2}$ of

$$
\begin{aligned}
\int_{R^{*}}\left(a_{i \beta} U_{x}^{\beta}+b_{i \beta} U_{y}^{\beta}+d_{i \beta} u^{\beta}\right) d y- & \left(b_{\beta i} U_{x}^{\beta}+c_{i \beta} U_{y}^{\beta}+e_{i \beta} u^{\beta}\right) d x \\
= & \iint_{R}\left(d_{\beta i} u_{x}^{\beta}+e_{\beta i} u_{y}^{\beta}+f_{i \beta} u^{\beta}\right) d x d y .
\end{aligned}
$$

It can be shown that $T u$ is completely continuous over $\mathfrak{B}_{2}$ (not $\mathfrak{P}_{2}$ unless $\Delta$ is restricted) and even carries any function $u$ in $\mathfrak{B}_{2}$ into a function $U$ which is continuous on $\Delta$ and. can be bounded as in (15.2), the $\lambda$ there being any number less than $\frac{1}{2} \mu$ ( $\mu$ in (18.4) and the $M$ depending on the norm of $u$ and of the operator $T$ ). Then from the Riesz theory [23] of generalized integral equations, we can conclude that the transformation

$$
u-\rho T u=\phi
$$

has a linear inverse if $\rho$ is not one of a set of isolated characteristic values. If $\rho$ is not a characteristic value, we can solve the equations (18.1), with the parameter $\rho$ introduced as a multiplier of all the $d_{i j}, e_{i j}$, or $f_{i j}$, as follows: First let $\phi$ be the solution of (18.1) with $\rho=0$ and then solve (21.1) for $u$ in terms of $\phi$; the function $u$ so obtained is immediately seen to be the desired solution. In doing this, the $g_{i}$ and $k_{i}$ may be merely functions of class $L_{2}$ on $\Delta$, but we assume that the $d_{i j}, e_{i j}, f_{i j}$, and $l_{i}$ satisfy (18.4). As in $\$ 20$, if $g$ and $k$ satisfy (20.1) at a point $P$, we may draw similar conclusions, where the bound for $|\bar{u}(P)|$ given in (15.2) depends only on the quantities $m, M, P, \mu, T$, $\pi, \delta, a$, the norm of $T$, and the norm of the inverse operator of (21.1), $\delta$ and $a$ having their significance in $\$ 15$.

22. Let us now suppose that $\rho$ is not a characteristic value and let $P_{0}$ be any point in $\Delta$. Let us consider the solution $u$ of (18.1) where $l=0$ and $g$ and $k$ are in $L_{2}$ on $\Delta-C\left(P_{0}, \sigma\right)$ and zero in $C\left(P_{0}, \sigma\right)$; such functions satisfy any condition of the type (20.1) for circles $C\left(P_{0}, r\right)$. Clearly the value of $\bar{u}\left(P_{0}\right)$ is a linear functional defined on the space $L_{2}\left[\Delta-C\left(P_{0}, \sigma\right)\right]$ for each $\sigma>0$. We therefore conclude [24] the existence of functions $\Delta_{1}^{i j}\left(x_{0}, y_{0} ; x, y\right)$ and $\Delta_{2}^{i j}\left(x_{0}, y_{0} ; x, y\right)$ such that

$$
\bar{u}^{i}\left(x_{0}, y_{0}\right)=\iint_{\Delta}\left(\Delta_{1}^{i \beta} g_{\beta}+\Delta_{2}^{i \beta} k_{\beta}\right) d x d y .
$$

From the way these $\Delta_{k}^{i j}$ arise, we can conclude that there exist func- 
tions $G^{i j}\left(x_{0}, y_{0} ; x, y\right)$ of class $\mathfrak{B}_{1}^{\prime}$ over $\Delta$ and of class $\mathfrak{P}_{2}^{\prime \prime}$ on $\Delta-C\left(P_{0}, \sigma\right)$ for each $\sigma>0$ such that $G_{x}^{i j}=\Delta_{1}^{i j}, G_{y}^{i j}=\Delta_{2}^{i j}$. It can also be shown that the $G^{i j}$ possess other properties which lead us to call them the Green's matrix for (18.1). Moreover if the $g$ and $k$ satisfy (20.1) for every $P$ and $r$, we conclude that the continuous representative $\bar{u}$ of the solution $u$ of (18.1) in $\mathfrak{B}_{2}$ is represented everywhere by

$$
\bar{u}^{i}\left(x_{0}, y_{0}\right)=\iint_{\Delta}\left(G_{x}^{i \beta} g_{\beta}+G_{y}^{i \beta} k_{\beta}+G^{i \beta} l_{\beta}\right) d x d y, \quad i=1, \cdots, N
$$

and $\bar{u}^{i}$ satisfies a uniform Hölder condition on any region $H$ with $\bar{H}$ interior to $\Delta$, this Hölder condition depending only on the distance of $\bar{H}$ from $\Delta^{*}$, the quantities $m, M, P, \mu, T, \pi, a$, and any upper bounds for $\delta$ and the norms $T$ and the inverse in (21.1).

23. Finally, if $(u, v, V)$ is any solution of (18.6) on a circle $C\left(P_{1}, b\right)$ which satisfies (18.7) there, we can, if $\rho$ is not characteristic, express $u$ in a circle $C\left(P_{1}, c\right)$ with $c<b$ in the form

$$
\begin{array}{r}
\dot{u}^{i}\left(x_{0}, y_{0}\right)=\iint_{C\left(P_{1}, b\right)-C\left(P_{1}, d\right)}\left(G_{x}^{i \beta} g_{\beta}+G_{y}^{i \beta} k_{\beta}+G^{i \beta} l_{\beta}\right) d x d y, \\
\left(x_{0}, y_{0}\right) \varepsilon C\left(P_{1}, c\right), c<d<b,
\end{array}
$$

in which

$$
\begin{gathered}
g_{i}=A_{i \beta}^{1} u^{\beta}+B_{i \beta}^{1} v^{\beta}+C_{i \beta}^{1} V^{\beta}, \quad k_{i}=A_{i \beta}^{2} u^{\beta}+B_{i \beta}^{2} v^{\beta}+C_{i \beta}^{2} V^{\beta}, \\
l_{i}=A_{i \beta}^{3} u^{\beta}+B_{i \beta}^{3} v^{\beta}+C_{i \beta}^{3} V^{\beta}
\end{gathered}
$$

where the $A_{i j}^{k}, B_{i j}^{k}$, and $C_{i j}^{k}$ are bounded and measurable and independent of $\left(x_{0}, y_{0}\right)$. Also there exists a number $\delta_{0}>0$ which depends only on $m, M, P$, and $\mu$ such that if $\delta \leqq \delta_{0}$, the norm of $T$ is less than or equal to $\frac{1}{2}$ and the norm of the inverse of (21.1) is less than or equal to 2 for all $\rho \leqq 1$. Thus $\rho=1$ is surely not a characteristic value if $b \leqq \frac{1}{2} \delta_{0}$ and the representation (23.1) satisfies a uniform Hölder condition in $C\left(P_{1}, c\right)$ which depends only on $m, M, P, \mu, Q, \nu$ (of (18.7)), and $b-c$, if $b \leqq \delta_{0}$. Thus Theorem 19.1 then follows, for, if the solution is given on $\Delta$ and $H$ is any subregion with $\bar{H} \subset \Delta$, we may choose $b$ and $c$ so that $\bar{H}$ can be covered by a finite number of circles $C\left(P_{i}, c\right)$ where $C\left(P_{i}, b\right)$ belongs to $\Delta$ for each $i$.

\section{BIBLIOGRAPHY}

1. The new results stated in this address (and some other results) are to be found in the following eight papers, all but the first of which are by the author. The 
first two appear in the Duke Mathematical Journal, vol. 6 (1940), pp. 170-186, 187215 , and the other six will probably appear shortly in the University of California Mathematical Series.

(i) Functions of several variables and absolute continuity I, by J. W. Calkin.

(ii) and (iii) the same, parts II and III.

(iv) The existence of functions minimizing certain multiple integrals.

(v) Lemmas in potential theory.

(vi) A general form of Haar's lemma.

(vii) Elliptic linear self adjoint systems of partial differential equations in two independent variables.

(viii) The differentiability of functions minimizing certain double integrals.

Numbers (iv) and (viii) have been presented to the Society (February 26, 1938, and April 16, 1938), number (iv) under the title The existence of solutions of certain minimum problems for multiple integrals.

2. L. Tonelli, Sur la semi-continuité des intégrals doubles du calcul des variations, Acta Mathematica, vol. 53 (1929), pp. 325-346; and L'estremo assoluto degli integrali doppi, Annali della Scuola Normale Superiore di Pisa, (2), vol. 2 (1933), pp. 89-130.

3. E. J. McShane, Integrals over surfaces in parametric form, Annals of Mathematics, (2), vol. 34 (1933), pp. 815-838; and Existence theorems for double integral problems of the calculus of variations, Transactions of this Society, vol. 38 (1935), pp. 549-563.

4. E. Hopf, Zum analytischen Charakter der Lösungen regulärer zweidimensionaler Variationsprobleme, Mathematische Zeitschrift, vol. 30, pp. 404-413.

5. C. B. Morrey, Jr., On the solutions of quasi-linear elliptic partial differential equations, Transactions of this Society, vol. 43 (1938), pp. 126-166.

6. G. C. Evans, Fundamental points of potential theory, Rice Institute Pamphlets, vol. 7, no. 4 (1920), pp. 252-329, particularly pp. 274, 275 . We shall refer to this paper by the letter $P$.

7. G. C. Evans, Complements of potential theory, part II, American Journal of Mathematics, vol. 55 (1933), pp. 29-49, especially pp. 42-46.

8. L. Tonelli, Sulla quadratura delle superficie, Atti della Reale Accademia dei Lincei, (6), vol. 3 (1926), pp. 633-638.

9. See, for instance, de la Vallée Poussin, Intégrales de Lebesgue, Fonctions d'Ensemble, Classes de Baire, Paris, 1916, pp. 59-61.

10. S. Banach, Théorie des Opérations Linéaires, Warsaw, 1932, chap. 4. We shall refer to this book as "Banach."

11. H. Rademacher, Über partielle und totale Differenzierbarkeit von Funktionen mehrerer Variabeln, und über die Transformation der Doppelintegrale, Mathematische Annalen, vol. 79 (1918), pp. 340-359.

12. See P [6], p. 282.

13. S. Saks, On the surfaces without tangent planes, Annals of Mathematics, (2), vol. 34 (1933), pp. 114-124.

14. R. Courant and D. Hilbert, Methoden der mathematischen Physik, Berlin, 1937, vol. 2, chap. 7, $\$ 1$.

15. Banach, chap 9.

16. Banach, p. 23.

17. R. Courant, Plateau's problem and Dirichlet's principle, Annals of Mathematics, (2), vol. 38 (1937), pp. 679-724.

18. A. Haar, Über die Variation der Doppelintegrale, Journal für die reine und angewandte Mathematik, vol. 149 (1919), pp. 1-18; and Über das Plateausche Prob- 
lem, Mathematische Annalen, vol. 97 (1926-1927), pp. 124-158, particularly pp. 146151.

19. L. Lichtenstein, Über den analytischen Charakter der Lösungen regulärer zweidimensionaler Variationsprobleme, Bulletin de l'Académie des Sciences de Cracovie, Classe des Sciences Mathématiques et Naturelles, (A), December, 1912, pp. 915-941.

20. S. Bochner, Harmonic surfaces in Riemann metric, this Bulletin, vol. 45 (1939), p. 832.

21. J. Leray and J. Schauder, Topologie et équations fonctionelles, Annales Scientifiques de L'École Normale Supérieure, (3), vol. 51 (1934), pp. 45-78.

22. C. B. Morrey, Jr., The problem of Plateau on a Riemannian manifold, this Bulletin, abstract 45-9-350.

23. Banach, chap. 10. For the original see F. Riesz, Über lineare Funktionalgleichungen, Acta Mathematica, vol. 41 (1916), pp. 71-98.

24. Banach, chap. 4.

University of CALIFornia 